\title{
Trivium
}

Revue franco-allemande de sciences humaines et sociales - Deutsch-französische Zeitschrift für Geistesund Sozialwissenschaften

28 | 2018

Capitalisme

\section{Die Anerkennung des Unternehmens im Recht: für eine »Société à Mission«}

\section{Armand Hatchuel et Blanche Segrestin}

Traducteur : Katrin Heydenreich

\section{CpenEdition}

Journals

Édition électronique

URL : http://journals.openedition.org/trivium/5959

DOI : 10.4000/trivium.5959

ISBN : 1963-1820

ISSN : 1963-1820

\section{Éditeur}

Les éditions de la Maison des sciences de l'Homme

\section{Référence électronique}

Armand Hatchuel und Blanche Segrestin, « Die Anerkennung des Unternehmens im Recht: für eine » Société à Mission« », Trivium [Online], 28 | 2018, online erschienen am 30 Juli 2018, abgerufen am 08 September 2020. URL : http://journals.openedition.org/trivium/5959 ; DOI : https://doi.org/10.4000/ trivium.5959

Ce document a été généré automatiquement le 8 septembre 2020.

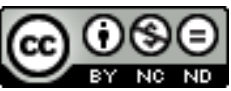

Les contenus des la revue Trivium sont mis à disposition selon les termes de la Licence Creative Commons Attribution - Pas d'Utilisation Commerciale - Pas de Modification 4.0 International. 


\title{
Die Anerkennung des Unternehmens im Recht: für eine » Société à Mission«
}

\author{
Armand Hatchuel et Blanche Segrestin \\ Traduction : Katrin Heydenreich
}

\section{NOTE DE L'ÉDITEUR}

Wir danken Blanche Segrestin und Armand Hatchuel für die freundliche Genehmigung, diesen Artikel in deutscher Übersetzung und in der französischen Originalfassung zu publizieren.

Nous remercions Blanche Segrestin et Armand Hatchuel de nous avoir accordé l'autorisation de traduire ce texte pour le présent numéro ainsi que d'y publier le texte original.

1 In Zeiten wirtschaftlicher und gesellschaftlicher Krisen wird von den Unternehmen nicht nur erwartet, dass sie neue Ressourcen entwickeln, um wettbewerbsfähig zu bleiben, sondern auch, dass sie neue Wege einschlagen, um den sozialen und ökologischen Herausforderungen, vor denen unsere Gesellschaften stehen, zu begegnen.

2 Voraussetzung für ein gerechtes und nachhaltiges Wachstum ist dabei nicht zuletzt die Innovationsfähigkeit der Unternehmen: Schaffung dauerhafter und qualitativer Arbeitsplätze, Entwicklung von Lösungen für die Energiewende, Entwicklung umweltfreundlicher Technologien, Erprobung neuer Lebensmodelle ... Doch sind die Unternehmen, so wie sie heute definiert und gemanagt werden, überhaupt in der Lage, sich derartigen Herausforderungen $\mathrm{zu}$ stellen? Tatsache ist doch, dass Großunternehmen ihre Gewinne heute hauptsächlich für Dividendenausschüttungen und Aktienrückkäufe verwenden, was die Finanzierung von Projekten von gemeinschaftlichem Interesse stark beeinträchtigt. ${ }^{1}$ Die gegenwärtige Krise zeigt, dass 
nicht mehr davon ausgegangen werden kann, dass Unternehmen, die gesellschaftliche Verantwortung übernehmen wollen, dazu auch den nötigen Handlungsspielraum besitzen.

3 Bei der Untersuchung der Gründe für diese Krise wird erkennbar, dass der rechtliche Rahmen den Unternehmen keine völlige Handlungsfreiheit lässt. Nicht, dass er zu bestimmten strategischen Optionen drängen würde, sondern weil er eine auf die Aktionäre bezogene Konzeption des Unternehmens und ein ebensolches Managementmodell und, damit einhergehend, eine wachsende und schwer einzudämmende Fokalisierung auf kurzfristige Rentabilität ermöglicht hat. Innovation, Langzeitperspektive und gesellschaftliche Verantwortung sind in diesem Kontext zwar nicht verschwunden, doch sie sind nicht mehr vorrangig. Darüber hinaus sind sie rechtlich nicht abgesichert, etwa im Falle eines Aktionärswechsels. ${ }^{2}$ Die von der École des Mines mit Unterstützung des Collège des Bernardins ${ }^{3}$ initiierten Arbeiten im Bereich Betriebswirtschaftslehre zeigen, dass es höchste Zeit ist, andere Leitlinien für Unternehmen wie auch Unternehmensführung zu entwickeln, um nicht nur aus der Finanzkrise, sondern auch aus der Krise in den Bereichen Forschung und Entwicklung (F\&E) und Innovation einen Ausweg zu finden. ${ }^{4}$

\section{Krise des Aktionärsunternehmens: ein rechtlicher Defekt}

4 Die gegenwärtige Wirtschaftskrise lässt sich nur verstehen, wenn man die in den 1980er Jahren eingeleitete tiefgreifende Umwälzung der Unternehmensführung ermisst. Die großen Unternehmen, die den Großteil der weltweiten Investitionen in F\&E bestreiten, mussten mit ansehen, wie ihr Management zunehmend dem Kriterium des Shareholder-Value unterworfen wurde. Es wurden Anreiz-, Entschädigungs- und Ratingdispositive entwickelt, um den Handlungsrahmen für die Unternehmensleitung festzustecken und, wie es die Agency-Theorie verlangt, ihre Entscheidungen und ihr Verhalten am Interesse der Aktionäre "auszurichten «. Die corporate governance hat sich als neue internationale Norm etabliert und sämtliche Managementkriterien bis hin zu den Buchführungsregeln verändert.

5 In diesem Wandel liegt der Grund für das erstaunliche Abdriften des Bankenmanagements, das 2008 zur Finanzkrise geführt hat, von der auch das gesamte Industrienetz, das vom Finanzmarkt abhängig ist, in Mitleidenschaft gezogen wurde. Projekte, die auf langfristige Innovationsentwicklungen abzielten, und erst recht solche, die andere Ziele als die reine kurzfristige Rentabilität verfolgten (z. B. die Entwicklung umweltfreundlicher Technologien), wurden davon am stärksten betroffen. Ein derartiger Wandel hat so manchen überrascht, wurde Management doch herkömmlicherweise als etwas angesehen, das zum Nutzen einer breiten Interessengemeinschaft auf die langfristige Entwicklung des Unternehmens hinarbeitet. Diese industrie- und fortschrittorientierte Konzeption des Unternehmens und seines Managements, die übrigens mit der relativen Schwäche des Finanzsektors vereinbar war, hatte bis zu den 1990er Jahren Gültigkeit. Doch dann wurde sie abgelöst von neuen ökonomischen Grundsätzen, die das Unternehmen vor allem als eine Gesamtheit an finanziellen Aktiva und die Unternehmensführung vor allem als "Agenten" der Aktionäre verstehen. Diese bemerkenswerte Regression in den 
Grundsätzen hatte tiefgreifende wirtschaftliche und soziale Auswirkungen, und sie konnte nur deshalb geschehen, weil das Unternehmen keinerlei rechtlichen Schutz hat.

Das Unternehmen als Handlungsgemeinschaft und Vektor für die kollektive Schaffung von Reichtum hat keine rechtliche Grundlage; im [französischen] Recht gibt es das Unternehmen (entreprise) nicht. ${ }^{5}$ Es existiert lediglich die Handelsgesellschaft (société commerciale), d.h. ein Vertrag, der die verschiedenen Anteilseigner und die Unternehmensleitung bindet. In den Aktiengesellschaften sind allein die Aktionäre in der Lage, die Unternehmensleitung zu ernennen und zu kontrollieren. Wenn sie urteilen, dass die Managementstrategie nicht ihren Interessen entspricht, kann sie nichts davon abhalten, die Unternehmensleitung abzusetzen. In anderen Worten kann ein Unternehmen sehr wohl ehrgeizige Entwicklungsprojekte verfolgen wollen, doch diese sind letztendlich immer dem Wohlwollen des Gesellschafter-Kreises unterworfen. Einem Unternehmen droht jederzeit die Gefahr einer feindlichen Übernahme oder einer Änderung der Kontrollmehrheit. Es gibt weder im französischen noch im amerikanischen Gesellschaftsrecht ein Mittel, sich dagegen $\mathrm{zu}$ wehren, dass das Interesse eines Unternehmens auf seinen Shareholder-Value verkürzt wird, wenn die Aktionäre das so entschieden haben. Genauso wenig ist im Gesellschaftsrecht vorgesehen, die Aktionäre $\mathrm{zu}$ einem Engagement zu bewegen. Natürlich gibt es engagierte Anteilseigner, z. B. in Familienunternehmen, die bekanntlich oft in langfristige Projekte investiert sind. Doch letztendlich hängt das Engagement der Aktionäre immer von deren Wohlwollen ab: rechtlich gibt es keine Grundlage, es einzuklagen. Wie aber sollen Aktionäre sich langfristig und erst recht für gesellschaftliche und ökologische Ziele engagieren, wenn sie nicht einmal ihrem Unternehmen gegenüber zu Loyalität verpflichtet sind?

7 Es darf nicht verwundern, dass es den Unternehmen unter diesen Bedingungen schwerfällt, ihrer gesellschaftlichen Verantwortung (Steuerzahlungen, wie kürzlich gesehen, inbegriffen) nachzukommen, selbst wenn Corporate Social Responsability Normen wie ISO 26000 immer mehr an Bedeutung gewinnen. Auch dürfte es immer schwerer werden, vielgestaltige und innovative Projekte zu entwickeln, obwohl genau diese letztendlich die Wettbewerbsfähigkeit der Unternehmen ausmachen: Die Zahlen in den USA belegen auf frappierende Weise, dass in den Jahren vor der Finanzkrise 2008 die CEOs der Großunternehmen - zweifellos durch massive Stockoptions-Programme in die Falle gelockt - ihr Unternehmen eher dazu veranlasst haben, die eigenen Aktien zurückzukaufen als in Forschungs- und Entwicklungsprojekte zu investieren. ${ }^{6}$

8 Die Gefahren fehlender ethischer Grundsätze im Managementbereich müssen anerkannt werden und es gilt, über Mittel und Wege nachzudenken, wie die Rechtslücke bezüglich des Unternehmens geschlossen werden kann.

\section{Unternehmen: ein Auftrag, der weit mehr bedeutet als Gewinn}

Zunächst darf das Unternehmen nicht mit dem wirtschaftlichen Instrument des Kapitalismus verwechselt werden, d.h. es darf insbesondere nicht mit der Handelsgesellschaft (société commerciale) gleichgesetzt werden. Das moderne Unternehmen ist weder auf die Handelsgesellschaft noch auf einen nach Gewinnmaximierung strebenden wirtschaftlichen Akteur reduzierbar. Um zu verstehen inwiefern sich das moderne Unternehmen von der Handelsaktivität unterscheidet, 
muss man sich vergegenwärtigen, dass es erst zum Ende des 19. Jahrhunderts entstanden ist, also sehr spät in Bezug auf die Geschichte des Kapitalismus. Es entstand erst, als die wissenschaftlichen und technischen Entwicklungen eine Neuorganisierung der Autoritäts- und Arbeitsbeziehungen verlangten, die vorher durch den Markt geregelt waren. Zur Schaffung von Neuem, zur Innovation, bedurfte es Kompetenzen, die nicht immer bereits verfügbar waren. Es mussten kollektive Aus- oder Weiterbildungen auf den Weg gebracht, neue Berufe, Methoden und Organisationsformen entwickelt werden. Genau das war die Aufgabe der Ingenieure, aus deren Reihen die neuen Unternehmensführer auch oft stammten. Ende des 19. Jahrhunderts entwickelten sie eine neue, auf Fachkenntnis gegründete Führungsart. Die Dynamik gemeinschaftlichen Schaffens ging auch einher mit einem neuen Verhältnis zur Arbeit, das durch eine mit der Einführung des Arbeitsvertrags verbundene Unterordnung [gegenüber dem Arbeitgeber] und neuartige Vertretungsmechanismen der Beschäftigten gekennzeichnet war. Im Zuge dessen entstand eine neue Autoritätsfigur: der chef d'entreprise, der Unternehmensführer, der die Hoffnung auf eine »neutrale Technokratie« im Dienst kollektiven Fortschritts und befriedeter sozialer Beziehungen verkörperte.

Das moderne Unternehmen unterscheidet sich von den klassischen Organisationsformen des Kapitalismus, und insbesondere von den alten Handelskompanien, in mindestens vier Punkten:

11 1. Das Unternehmen definiert sich durch seinen Auftrag kollektiven Schaffens. Um von "Unternehmen« sprechen zu können, genügt es weder, jemanden einzustellen, noch eine lukrative Handelsaktivität zu verfolgen; es braucht ein Projekt zur Entwicklung neuer Handlungskapazitäten.

2. Unternehmensführer sind keine "Gesellschaftsmandatare" (mandataires sociaux) im Sinne des Gesellschaftsrechts. Vielmehr werden sie für ihre Fähigkeit gewählt, neue Anwendungen der Unternehmensressourcen $\mathrm{zu}$ entwickeln und ein Unternehmensprojekt auszuführen. Ihr Status sollte daher ausdrücklich im Sinne einer "Ermächtigung" definiert werden (wie beim Schiffskapitän), und zwar einer Ermächtigung durch alle Personen, die sich in dem gemeinschaftlichen Projekt engagieren und somit ihr eigenes "Handlungspotenzial", d.h. ihre Entwicklungskapazitäten und ihr kreatives Potenzial, der Unternehmensleitung anvertrauen.

13 3. Das Unternehmen, sein Wirkungskreis, beschränkt sich selbstverständlich nicht auf die Aktionäre, sondern umfasst auch die Beschäftigten und mitunter noch weitere Interessenten. Die Grenze ist von einem Unternehmen zum nächsten anders gezogen, doch ein Prinzip scheint sich durchzusetzen: Unter allen Stakeholdern, die von einer Managemententscheidung möglicherweise betroffen sind, sollten nur diejenigen, die sich im Unternehmen engagieren, d. h. die auf ihre Autonomie verzichten, indem sie eine einzige Führungsautorität anerkennen, an der Ernennung der Unternehmensleitung teilhaben dürfen.

4. Ein Solidaritätsprinzip im Fall von »Havarien«: Managemententscheidungen können sich auf die Potenziale aller im Unternehmen engagierten Parteien auswirken. Manche Entscheidungen, wie Sozialpläne oder Umstrukturierungen, werden zum Nachteil der Beschäftigten im Namen des "gemeinsamen Gutes", das das Unternehmen darstellt, getroffen. In solchen Situationen sollte man den im Seerecht festgelegten Grundsatz der "großen Haverei« als Solidaritätsmodell im Unternehmen heranziehen. Diesem 
Grundsatz zufolge müssen Schäden, die auf Anordnung des Schiffskapitäns verursacht wurden, um die Expedition zu retten, von allen Beteiligten getragen werden, die ein Interesse daran haben, dass das Schiff gerettet wird. ${ }^{7}$ Müsste analog dazu ein von einem Aktionär realisierter Mehrwert nicht zum Teil an das gesamte Kollektiv zurückgehen? Müssten zugunsten des zukünftigen Wohls des Unternehmens veranlasste betriebliche Entlassungen oder kollektive Lohnsenkungen nicht über die gesetzlichen Entschädigungen hinaus kompensiert werden (z. B. über eine Austeilung von Aktien)? Dies würde einfache Gerechtigkeitskriterien für künftige Vereinbarungen zur Steigerung der Wettbewerbsfähigkeit vorgeben.

Alle genannten Prinzipien hätten jedoch mehr Durchsetzungskraft, wenn sie rechtlich festgeschrieben wären. Die klassische Aktiengesellschaft lässt zu viele Ausartungen zu. Es ist dringend erforderlich, über neue Gesellschaftsformen nachzudenken.

\section{Ein Vorschlag: die »Société à Mission«}

Die Notwendigkeit, das Recht weiterzuentwickeln, um die Herausbildung innovativer und verantwortlicher Unternehmen anzuregen, hat in mehreren Ländern bereits zu originellen Gesellschaftsformen geführt. Sowohl in Europa als auch jenseits des Atlantiks wurden neue Rechtsformen geschaffen, die wirtschaftliche Aktivität mit gesellschaftlich und ökologisch verantwortlichen Projekten vereinen wollen. $\mathrm{Zu}$ nennen wären hier z. B. die - bald auch in Luxemburg übernommene - belgische Société à Finalité Sociale, die britische Community Interest Company, die französische Société Coopérative d'Intérêt Collectif oder die Benefit oder Flexible Purpose Corporation in den USA.

Für Frankreich schlagen wir eine rechtliche Neuerung vor, die von demselben Geist getragen wird: die Einführung der "Société à Mission«, "Gesellschaft mit Auftrag«. Sie würde dazu beitragen, den gesellschaftlichen Zweck zu erweitern, indem in die Unternehmensstatuten aufgenommen würde, dass das Unternehmen abgesehen von der Gewinnerzeugung weitere Ziele, z. B. den Erhalt von Arbeitsplätzen, die Aufgabe umweltverschmutzender Technologien, ein geteiltes Management usw. verfolgt. Vor allem würde ein derartiger Status dem Unternehmensführer einen offeneren Handlungsrahmen bieten und dessen Visions- und Orientierungskompetenz wiederherstellen. Er könnte den Unternehmen der Économie Sociale et Solidaire [Sozialwirtschaft] dienen, ist jedoch generell in erster Linie für alle gewinnorientierten Unternehmen gedacht, die sich als sozial verantwortlich und auf gesellschaftlichen Fortschritt bedacht verstehen.

In der Praxis könnte das Recht für eine herkömmliche Gesellschaft - also Aktiengesellschaft (Société Anonyme, SA), vereinfachte Aktiengesellschaft (Société anonyme simplifiée, SAS), Arbeiterproduktivgenossenschaft (Société Coopérative et Participative, SCOP) usw. -, die Möglichkeit schaffen, einen Auftrag in ihre Statuten einzuschreiben, gleich, ob dieser nun einen spezifisch sozialen oder ökologischen Zweck (z. B. Eingliederung) verfolgt oder gesellschaftliche bzw. ökologische Ziele, die an eine wirtschaftliche Aktivität gekoppelt sind (z. B. Erhalt von Arbeitsplätzen). Damit wäre der Unternehmensauftrag für sämtliche Aktionäre bindend (und nicht nur für die Unternehmensführung), und darüber hinaus würde er auch einklagbar, und zwar, wenn die Statuten es so vorsehen, nicht nur von den Aktionären, sondern auch von Seiten anderer Parteien. Die Statuten müssten demnach sowohl eine Prozedur zur 
Billigung des Unternehmensauftrags als auch eine Prozedur zur Beurteilung des Managements vorsehen. In der Praxis schlagen wir Folgendes vor: ${ }^{8}$

- Die Definition oder Überarbeitung des Unternehmensauftrags muss von einer Mehrheit von zwei Dritteln der Anteilseigner befürwortet werden und ebenso vom Betriebsrat bzw. den Arbeitnehmervertretern.

- Die Evaluierung des Managements in Bezug auf den Unternehmensauftrag muss einem vom Aufsichtsrat abgegrenzten »Missionsrat« anvertraut werden. Dieser umfasst nur einen Teil aller Stakeholder, nämlich diejenigen, die vom Unternehmensauftrag betroffen sind: $d . h$. die Interessengruppen, gegenüber denen das Unternehmen sich engagiert, aber auch die interessierten Parteien, die kompetent sind, die Einhaltung des Engagements zu beurteilen.

Trotz seiner Einfachheit könnte dieser Vorschlag viele positive Auswirkungen haben. Er erkennt das Unternehmen im Recht an und stellt eine Definition des Unternehmens wieder her, die kohärent mit den historischen und theoretischen Grundlegungen des Unternehmens ist, d. h. seinem Auftrag kollektiver Schöpfung. Weiterhin würde er den Anstrengungen im Bereich der gesellschaftlichen Verantwortung (Corporate Social Responsability) mehr Glaubwürdigkeit verleihen, weil die vom Unternehmen eingegangenen Engagements eingeklagt werden könnten. Er würde Unternehmensprojekte ermöglichen, die die Voraussetzungen für Innovation und wirtschaftliche Effizienz nachhaltig mit gesellschaftlichen und ökologischen Forderungen in Einklang bringen möchten. Schließlich würde er unternehmerische Freiheit und Allgemeinwohl versöhnen. Und wie jeder Rechtsakt hätte der Vorschlag auch eine pädagogische Wirkung: Er würde allen in Erinnerung rufen, dass den diversen Formen ökonomischen Handelns nichts Natürliches anhaftet und dass sie sich mit den gesellschaftlichen Bedürfnissen und Werten weiterentwickeln müssen.

\section{BIBLIOGRAPHIE}

Auvray, T. / Dallery, T. / Rigot, S. (2016): L'entreprise liquidée. La finance contre l'investissement, Paris: Michalon.

Favereau, O. (2014): Entreprises: la grande déformation, Paris: Parole et Silence, Collège des Bernardins.

Gutiérrez, G. N. / Philippon, T. (2016): Investment-less Growth: An Empirical Investigation. NBER Working Paper Series, Nr. 22897.

Lazonick, W. (2009): »The explosion of executive pay and the erosion of American prosperity«, Entreprises et Histoire, Nr. 57, S. 141-164.

Lazonick, W. (2014): »Profits Without Prosperity«, Harvard Business Review, Nr. 92, S. 46-55.

Robé, J.-P. (1999): L'entreprise et le droit, Paris: puf.

Roger, B. (Hg.) (2012): L'entreprise, formes de la propriété et responsabilités sociales, Paris: Parole et Silence / Lethielleux. 
Sandberg, J. (2011): „Socially Responsible Investment and Fiduciary Duty: Putting the Freshfields Report into Perspective«, Journal of Business Ethics, Nr. 101, S. 143-162.

Segrestin, B. / Hatchuel, A. (2011): »Autorité de gestion et avaries communes: pour un complément du droit de l'entreprise?«, Finance Contrôle Stratégie, Nr. 14, S. 9-36.

Segrestin, B. / Hatchuel, A. (2012): Refonder l'entreprise, Paris: Seuil (République des idées).

Segrestin, B. / Levillain, K. / Vernac, S. / Hatchuel, A. (Hg.) (2015): La société à objet social etendu: un nouveau statut pour l'entreprise, Paris: Presses des Mines.

\section{NOTES}

1. Lazonick (2014); Auvray et al. (2016); Gutiérrez / Philippon (2016).

2. Sandberg (2011)

3. Im Rahmen des vom Collège des Bernardins initiierten interdisziplinären Forschungsprogramms zum Unternehmen (2009-2018). Vgl. Roger (2012); Favereau (2014).

4. In diesem Aufsatz fassen wir die Thesen unseres Buches Segrestin / Hatchuel (2012) zusammen. Es wurde 2013 vom Berufsverband Syntec und der Société Française de Management (SFM) als bestes Buch für akademische Management-Forschung ausgezeichnet.

5. Robé (1999).

6. Lazonick (2009).

7. Segrestin / Hatchuel (2011).

8. Segrestin et al. (2015)

\section{INDEX}

Mots-clés : entreprise, mission, résponsabilité sociale d'entreprise, droit

Schlüsselwörter : Unternehmen, Mission, corporate social responsibility, Recht

\section{AUTEURS}

\section{ARMAND HATCHUEL}

Armand Hatchuel ist Professor an der Hochschule Mines ParisTech. Nähere Informationen finden Sie hier.

\section{BLANCHE SEGRESTIN}

Blanche Segrestin ist Professorin an der Hochschule Mines ParisTech. Nähere Informationen finden Sie hier. 